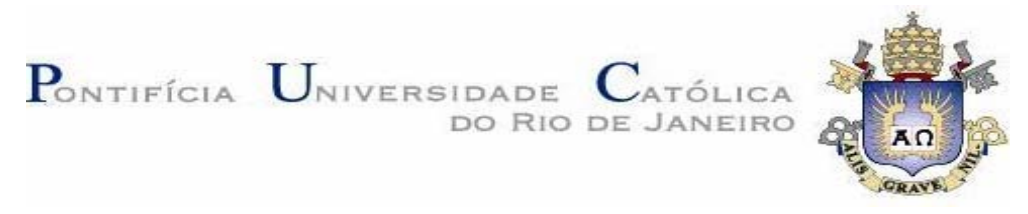

Wagner Felippe Pacheco

\author{
Desenvolvimento e comparação de \\ métodos voltamétricos para a \\ determinação de ciclofenil e primaquina \\ em medicamentos e em urina
}

Dissertação de Mestrado

Dissertação apresentada como requisito parcial para obtenção do título de Mestre pelo Programa de PósGraduação em Química da PUC-Rio.

Orientador: Ricardo Queiroz Aucélio 


\section{Desenvolvimento e comparação de métodos voltamétricos para a determinação de ciclofenil e primaquina em medicamentos e em urina}

Dissertação apresentada como requisito parcial para obtenção do título de Mestre pelo Programa de PósGraduação em Química da PUC-Rio. Aprovada pela Comissão Examinadora abaixo assinada.

Prof. Ricardo Queiroz Aucélio Orientador Departamento de Química-Puc-Rio

Profa. Roberta Lourenço Ziolli Departamento de Química-Puc-Rio

Prof. Pércio A. M. Farias Departamento de Química-Puc-Rio

Profa. Andréa Fernandes Arruda Instituto de Química-UnB

Prof. José Eugenio Leal Coordenador(a) Setorial do Centro Técnico Científico-PUC-Rio 
Todos os direitos reservados. É proibida a reprodução total ou parcial do trabalho sem autorização da universidade, do autor e do orientador.

\section{Wagner Felippe Pacheco}

Graduou-se em técnico metalúrgico pela Escola Técnica Federal do Espírito Santo (ETFES) em 1996. Entrou no curso de química da Universidade Federal do Espírito Santo (UFES) no ano de 1997, desenvolveu trabalhos de iniciação científica nos anos de 1998-2000 na área de química inorgânica. Formou-se em Bacharel e Licenciatura plena em química no ano de 2002.

Ficha Catalográfica

Pacheco, Wagner Felippe
Desenvolvimento e comparação de métodos
voltamétricos para a determinação de ciclofenil e
primaquina em medicamentos e em urina / Wagner Felippe
Pacheco ; orientador: Ricardo Queiroz Aucélio. - Rio de
Janeiro : PUC, Departamento de Química, 2004.
111 f. : il. ; 30 cm
Dissertação (mestrado) - Pontifícia Universidade
Inclui referências bibliográficas.
1. Química - Teses. 2. Ciclofenil. 3. Primaquina. 4.
Voltametria de pulso diferencial. 5. Voltametria de onda
quadrada. U. Urina. I. Aucélio, Ricardo Queiroz. II.
Pontifícia Universidade Católica do Rio de Janeiro.
Departamento de Química. III. Título

CDD: 540 
A Deus

A meus Pais, pela minha criação, pelo contínuo apoio, e por estarem sempre presentes em minha vida.

A Nathália, que mesmo à distância, continua sendo a razão da minha força, da minha alegria e da minha dedicação. 


\section{Agradecimentos}

A meu orientador, Ricardo Queiroz Aucélio. Pela segura orientação e pelo excelente convívio durante todo o mestrado.

A CAPES e a PUC-Rio, pelo suporte indispensável para a realização deste trabalho.

Ao professor Pércio Augusto Mardini Farias, pela disponibilização do aparelho utilizado durante este trabalho.

Ao departamento de farmacologia da UnB, pela cessão do ciclofenil.

Aos professores da Banca Examinadora.

Ao colega Carlos Eduardo Cardoso, que muito me ajudou no aprendizado da técnica e do manuseio do instrumento.

A Anselmo, por sua disponibilidade e sua boa vontade em ajudar sempre que necessário, além de seus importantes conselhos.

A técnica e amiga Sônia Gomes Bezerra, por sua colaboração na realização de experimentos.

A todos os meus colegas da pós graduação da PUC-Rio.

Á meus amigos de Vitória que também estão fazendo pós no Rio. Em especial, a Rachel de Oliveira Nasser e Geisamanda Pedrini Brandão, por toda a força que me deram quando eu precisei.

A meus amigos Renata, Nédio, Cláudia, Wilson e Bárbara. Por colocarem alegria em meu dia-a-dia na PUC-Rio. 


\section{Resumo}

Pacheco, Wagner. Desenvolvimento e comparação de métodos voltamétricos para a determinação de ciclofenil e primaquina em medicamentos e em urina. Rio de Janeiro, 2004. 111 pgs. Dissertação de Mestrado - Departamento de Química, Pontifícia Universidade Católica do Rio de Janeiro.

Neste trabalho foram desenvolvidas metodologias eletroanalíticas baseadas na voltametria adsortiva de redissolução catódica com varredura de potencial de onda quadrada e de pulso diferencial para a determinação do ciclofenil em urina e em formulações farmacêuticas, e na voltametria anódica com varredura de potencial de onda quadrada e de pulso diferencial para a determinação da primaquina em formulações farmacêuticas. Comparando-se as duas técnicas de aquisição, a voltametria de onda quadrada foi escolhida para realizar as determinações do ciclofenil em urina e em formulação farmacêutica por se mostrar, além de uma técnica mais rápida, maior sensíbilidade. Para a determinação da primaquina a melhor técnica foi à voltametria de pulso diferencial.

As condições experimentais que possibilitaram um melhor desempenho analítico em termos da obtenção do menor limite de detecção e maior reprodutibilidade das leituras foram otimizados. No caso do ciclofenil, o composto mostrou ser instável no meio aquoso e orgânico para determinação voltamétrica, com sistemática diminuição da corrente faradaica. Assim, fez-se uso das propriedades eletroquímicas de um derivado estável obtido pela derivatização fotoquímica do composto (o composto foi irradiado com irradiação UV por 45 minutos em um reator fotoquímico), a corrente máxima obtida apresentou um potencial a $-1,28 \mathrm{~V}$. As condições experimentais que possibilitaram este sinal foram obtidas com $30 \mathrm{~s}$ de tempo de deposição do analito sobre o eletrodo de mercúrio, solução Britton-Robbinson pH 9,0 como eletrólito de suporte, potencial de acumulação de $-0,9 \mathrm{~V}$, amplitude de $250 \mathrm{mV}$, incremento de potencial de 2,0 $\mathrm{mV}$ no modo de onda quadrada.

Desta forma foi obtido limite de detecção da ordem de $10^{-8} \mathrm{~mol} \mathrm{~L}^{-1}$, faixa linear dinâmica de 2 ordens de grandeza, condições estas que possibilitaram a determinação do composto tanto em formulações farmacêuticas (Menopax ${ }^{\circledR}$ ) como em amostra de urina enriquecida com o analito de interesse. Foram feitos 
testes com subtâncias concomitantes da formulação farmacêutica e constatou-se que não existia problema com interferência na análise nesse tipo de amostra. Nas determinações em urina não houve a necessidade de se fazer tratamento prévio da amostra, apenas a irradiação UV para estabilizar o composto, as análises foram realizadas com o método de adição de analito, de forma a se corrigir a interferência de matriz. Em ambos os casos os resultados obtidos se encontram na faixa de 93,6 a 106,5 \%, dentro da faixa de recuperação aceitável para este tipo de problema analítico segundo estabelecido pela Farmacopéia dos Estados Unidos da América.

Usou-se ainda os resultados provenientes das voltametrias de pulso diferencial, de voltametria de onda quadrada e da voltametria cíclica para se obter informações sobre o processo redox que ocorria no eletrodo de trabalho. Constatou-se que a reação é reversível, com um processo de controle adsortivo, sendo que apenas o reagente adsorve no eletrodo de trabalho, não ocorrendo adsorção do produto da reação de redução. Constatou-se também que o processo envolve a transferência de apenas 1 elétron, e que a reação não possuía contribuição cinética.

No caso da primaquina o sinal de redução ocorre em região anódica $(0,592$ V), foi portanto necessário utilizar um eletrodo de carbono para se poder fazer a determinação da primaquina. Visando a possibilidade do uso do eletrodo de mercúrio, tentou-se fazer uso da formação de derivados complexos da primaquina com vanádio, com cobre ou com íon iodeto (complexo de tranferência de carga), porém não foram observados sinal da reação redox na janela de potencial procurada. Nas condições experimentais otimizadas não se pode pre-concentrar o analito no eletrodo de trabalho.

Utilizando-se o eletrodo de carbono vítreo e a técnica de pulso diferencial, determinou-se a primaquina em formulações farmacêuticas com recuperação média de $101,7 \%$, e limite de detecção de $1,0 \times 10^{-6} \mathrm{~mol} \mathrm{~L}^{-1}$.

\section{Palavras-chave}

voltametria de pulso diferencial; voltametria de onda quadrada; ciclofenil; primaquina; urina 


\section{Abstract}

Pacheco, Wagner Felippe. Development and comparison of voltammetric methods for the determination of cyclofenil and primaquine in pharmaceutical formulations and in urine

Rio de Janeiro, 2004. 111 p. Dissertação de Mestrado - Departamento de Química, Pontifícia Universidade Católica do Rio de Janeiro.

In this work, square-wave and differential pulse voltammetric methods were developed for the determination of cyclofenil and primaquine in pharmaceutical formulations and in urine samples. The use of the square-wave acquisition technique was found to enable better sensitivity and faster analysis time compared to the differential pulse technique.

Experimental and instrumental conditions were optimized to allow the best analytical performance in terms of limit of detection and repeatability of the readings. In the case of cyclofenil, its unstable behavior in aqueous and organic solvents, with systematic decreasing of analyte current signal, makes impossible any voltammetric determination. As an alternative way, the electrochemical properties of a stable photochemical derivative of cyclofenil was used (the compound was irradiated with UV radiation for $45 \mathrm{~min}$ ) with maximum current at $-1,28 \mathrm{~V}$. This analyte photoderivative could also be accumulated in the working electrode. The experimental conditions that allowed the maximum current was a $30 \mathrm{~s}$ of deposition time at the mercury electrode, Britton-Robbinson $(\mathrm{pH} \mathrm{9,0)}$ supporting electrolyte, accumulation potential of $-0,9 \mathrm{~V}$, amplitude of pulse of $250 \mathrm{mV}$, scan increment $2,0 \mathrm{mV}$.

These optimized conditions allowed a limit of detection of $10^{-8} \mathrm{~mol} \mathrm{~L}^{-1}$ and dynamic linear range of 2 orders of magnitude to be achieved. These analytical figures of merit made possible the determination of cyclofenil either in a pharmaceutical formulation $\left(\operatorname{Menopax}{ }^{\circledR}\right)$ and in urine samples spiked with the analyte of interest. The potential interferences from concomitant substances used in the pharmaceutical formulation were also evaluated. For the analyte determination in urine, only UV irradiation of sample was necessary, in order to obtain stable cyclofenil derivative. The analyte addition method was used to analyze urine in order to minimize matrix interferences. Recovery results for the analysis of Menopax ${ }^{\circledR}$ and for the analysis of urine were between 96,5 and 107,6 
$\%$, within the acceptable recovery range established by the United States Pharmacopoeia.

Information concerning the analyte redox reaction and electrode processes was also obtained from differential pulse voltammetry, square-wave voltammetry and cyclic voltammetry. It was verified that the cyclofenil photoderivative eletrochemical reaction is reversible with adsorption of only the reagent on the surface of the electrode. The adsorption of the electrochemical reduction product does not occur. It was also verified that the process involves the transference of only one electron, and there is no kinetics contribution in the reaction.

In the case of the primaquine the analyte reduction occurs in anodic region $(0,592 \mathrm{~V})$, therefore, it was necessary the use the carbon glass electrode to allow the determination of this analyte. The pre-concentration of the analyte in the working carbon glass electrode was also not attained with the experimental conditions used.

Several attempts were made to make possible the use of the mercury electrode, including the formation of charge transfer complexes with iodine and complexation with vanadium. However no success was obtained.

Using the carbon glassy electrode and DPV technique the determination of primaquine in pharmaceutical formulations and urine was performed with average recovery of $101.7 \%$. Limit of detention of $1,0 \times 10^{-6} \mathrm{~mol} \mathrm{~L}^{-1}$ was obtained.

\section{Key-words}

differential pulse voltammetry; square-wave voltammetry; cyclofenil; primaquine; urine 


\section{Sumário}

1 Introdução

1.1 O ciclofenil e seu uso como substância de aumento ilícito do $\begin{array}{ll}\text { desempenho atlético } & 20\end{array}$

1.2 A primaquina e seu uso como antimalárico 22

$\begin{array}{ll}1.3 \text { Objetivos } & 27\end{array}$

2 Voltametria 28

2.1 Voltametria de varredura linear 38

2.2 Voltametria de pulso diferencial. 39

2.3 Voltametria de onda quadrada 41

2.4 Voltametria cíclica 43

2.5 Voltametria de redissolução. $\quad 46$

2.6 Voltametria adsortiva de redissolução (AdSV) 49

3 Materiais e métodos $\quad 50$

3.1. Reagentes 50

3.2. Instrumentação 52

3.2.1. Reator fotoquímico 52

3.2.2. Analisador polarográfico 53

3.2.3 Equipamentos auxiliares $\quad 55$

3.3 Metodologia de trabalho 56

3.3.1 Lavagem do material 56

3.3.2 Preparação de amostras e padrões 56

3.3.3 Tratamento fotoquímico 57

3.3.4 Formulações farmacêuticas $\quad 57$

3.3.5 Fluídos Biológicos $\quad 57$

3.3.6 Procedimento de "clean-up" com sulfato de amônio 57

3.3.7 Procedimento para a medida eletroquímica 58

4 Determinação do ciclofenil utilizando o fotoproduto eletroativo no EGPM. 
4.1 Estudos preliminares $\quad 59$

4.1.1 Estudo do eletrólito suporte. 59

4.1.2 Estudo do efeito da irradiação e do pH. 63

4.2 Voltametria de pulso diferencial (DPV) 66

4.2.1 Estudo do potencial de deposição 66

4.2.2 Estudo do tempo de deposição 68

4.2.3 Estudo da influência da amplitude 70

4.2.4 Estudo da influência da velocidade de varredura 72

4.2.5 Parâmetros analíticos de mérito para DPV 76

4.3 Voltametria de onda quadrada (SWV) 77

4.3.1 Estudo do potencial de deposição 77

4.3.2 Estudo da influência do incremento da varredura. 78

4.3.3 Estudo da influência da freqüência 81

4.3.4 Estudo da influência da amplitude 83

4.3.5 Parâmetros analíticos de mérito para SWV 84

4.4 Voltametria cíclica $\quad 85$

4.5 Determinação do ciclofenil utilizando o seu fotoproduto estável $\quad 88$

4.5.1 Determinação em formulações farmacêuticas 88

4.5.2 Determinação em urina. $\quad 90$

5 Determinação da primaquina em ECV. 91

5.1 Estudos preliminares $\quad 91$

5.2 Estudo do eletrólito suporte $\quad 94$

5.3 Estudo do potencial de deposição e do tempo de acumulação. 95

5.4 Voltametria de pulso diferencial 97

5.4.1 Estudo da influência da amplitude 97

5.4.2 Influência da velocidade de varredura. 99

5.5 Voltametria de onda quadrada. 100

5.6 Determinação da primaquina em formulações farmacêuticas. 101

6 Conclusão 103

7. Referências Bibliográficas 106 


\section{Lista de figuras}

$\begin{array}{ll}\text { Figura } 1 \text { - Ciclofenil } & 20\end{array}$

Figura 2 - Primaquina $\quad 23$

Figura 3 - Representação esquemática da dupla camada segundo a síntese de Stern dos modelos de Helmholtz e Gouy-chapman. 30

Figura 4 - Camada de difusão de Nernst. 35

Figura 5- Forma de aplicação do potencial para cada tipo de voltametria $\quad 37$

Figura 6- Representação esquemática da voltametria com varredura linear. $\quad 38$

Figura 7 - Sinais de excitação para voltametria de pulso diferencial 39

Figura 8 - Representação esquemática da voltametria de onda quadrada. $\quad 42$

Figura 9 - formas de aplicação do potencial para a voltametria cíclica: a) potencial com varredura linear e b) potencial do tipo escada. $\quad 45$

Figura 10 - Representação esquemática do reator fotoquímico utilizado. $\quad 52$

Figura 11 - Representação esquemática do potenciostato de três eletrodos, com loop de retorno. 53

Figura 12 - Faixa de potencial de trabalho para o eletrodo de carbono vítreo (ECV) e para o eletrodo de gota pendente de mercúrio (EGPM) nos eletrólitos suportes utilizados.

Figura 13 - Voltamogramas do ciclofenil em Britton-Robbinson $\mathrm{pH} 9$, demonstrando a diminuição da corrente faradaica como o tempo. O pico $2 \mathrm{U}$ é do ciclofenil, e o $1 \mathrm{U}$ é oxigênio dissolvido.

Figura 14 - Espectros de absorção molecular na região do uv-visível para A) ciclofenil semirradiação e B) ciclofenil irradiado. Condições: meio metanol/água $1: 1$, ciclofenil 5,0 x 10-5 $\mathrm{mol} \mathrm{L}^{-1}$.

Figura 15 - Influência do pH sobre o sinal de uma solução de ciclofenil. Condições experimentais: ciclofenil $1,0 \times 10^{-6} \mathrm{~mol} \mathrm{~L}^{-1}$; tempo de deposição $30 \mathrm{~s}$; incremento $10 \mathrm{mV}$; frequência $100 \mathrm{~Hz}$, amplitude $250 \mathrm{mV}$, modo de varredura de potencial SWV.

Figura 16 - Influência do potencial de deposição sobre o sinal eletroanalítico de uma solução de ciclofenil tratado com radiação UV. Condições experimentais: Ciclofenil 2,2 × $10^{-5} \mathrm{~mol} \mathrm{~L}^{-1}$ irradiado por $45 \mathrm{~min}$; tempo de 
deposição $30 \mathrm{~s}$; velocidade de varredura $10 \mathrm{mV} / \mathrm{s}$; amplitude $100 \mathrm{mV}$.

Figura 17 - Influência do tempo de deposição sobre o sinal de uma solução de ciclofenil irradiado. Condições experimentais: velocidade de varredura 10 $\mathrm{mV} / \mathrm{s}$; amplitude $=100 \mathrm{mV}$; potencial de deposição $-0,600 \mathrm{~V}$; concentração de ciclofenil $6.6 \times 10^{-6}$.

Figura 18 - Influência da amplitude sobre o sinal lido de uma solução de ciclofenil irradiado. Condições experimentais: concentração de ciclofenil 6.6 x $10^{-6} \mathrm{~mol} \mathrm{~L}{ }^{-1}$; velocidade de varredura $10 \mathrm{mV} . \mathrm{s}^{-1}$, tempo de deposição $30 \mathrm{~s}$; potencial de deposição $-0,600 \mathrm{~V}$.

Figura 19 - Largura do pico a meia altura em função da amplitude utilizada. 71 Figura 20 - Efeito da velocidade de varredura sobre a leitura de uma solução de ciclofenil irradiado. Condições: concentração de ciclofenil $1,35 \times 10^{-5} \mathrm{~mol} \mathrm{~L}^{-}$ 1, tempo de deposição de $30 \mathrm{~s}$ amplitude de $100 \mathrm{mV}$.

Figura 21 - Relação entre a corrente de pico e a velocidade de varredura. Condições: concentração de ciclofenil irradiado $1,35 \times 10^{-5} \mathrm{~mol} \mathrm{~L}^{-1}$, tempo de deposição de $30 \mathrm{~s}$ amplitude de $100 \mathrm{mV}$.

Figura 22 - Influência da velocidade de varredura sobre o potencial do pico. 74 Figura 23 - voltamograma no modo de pulso diferencial de solução irradiada de ciclofenil. Condições: concentração de ciclofenil $=1,35 \times 10^{-5} \mathrm{~mol} \mathrm{~L}^{-1}$, tempo de deposição $30 \mathrm{~s}$, velocidade de varredura $10 \mathrm{mV} / \mathrm{s}$.

Figura 24 - curva analítica para o modo de pulso diferencial nas condições otimizadas.

Figura 25- Efeito do potencial de deposição sobre a corrente lida de uma solução irradiada de ciclofenil. Condições experimentais: concentração de ciclofenil $1,0 \times 10^{-6} \mathrm{~mol} \mathrm{~L}^{-1}$, tempo de deposição $30 \mathrm{~s}$; frequência $100 \mathrm{~Hz}$; incremento $=10 \mathrm{mV}$.

Figura 26 - Efeito do incremento da varredura para a leitura de uma solução de ciclofenil. Condições experimentais: concentração de ciclofenil 6,9 X $10^{-6}$ mol L $\mathrm{L}^{-1}$ tempo de deposição de $30 \mathrm{~s}$, amplitude de potencial de $250 \mathrm{mV}$, frequência de $100 \mathrm{~Hz}$.

Figura 27 - Voltamogramas da mesma solução de ciclofenil, com incremento de (A) 2 e (B) $10 \mathrm{mV}$.

Figura 28- Relações experimentais obtidas entre a corrente do pico e a raíz 
quadrada da frequência de aplicação do pulso de potencial para uma solução de ciclofenil. Condições experimentais: concentração de ciclofenil 6,88 x 10${ }^{6} \mathrm{~mol} \mathrm{~L}^{-1}$, tempo de deposição de $30 \mathrm{~s}$, incremento de varredura de $2 \mathrm{mV} .81$ Figura 29- Efeito da amplitude para uma solução irradiada de ciclofenil. Condições experimentais: tempo de deposição $30 \mathrm{~s}$; frequência $100 \mathrm{~Hz}$; incremento $10 \mathrm{mV}$; concentração de ciclofenil $3,0 \times 10^{-6} \mathrm{~mol} \mathrm{~L}^{-1}$.

Figura 30- curva analítica para determinação de ciclofenil nas condições otimizadas para onda quadrada. $\quad 84$

Figura 31 - voltamograma cíclico para uma solução irradiada de ciclofenil 85 Figura 32- Relação entre a raíz quadrada da velocidade de varredura e a corrente do pico.

Figura 33 - Comparação ente as curvas analíticas obtidas no modo DPV e SWV.

88

Figura 34 - Influência do pH sobre o sinal de oxidação da primaquina. Condições: Primaquina 4,2 $\times 10^{-5} \mathrm{~mol} \mathrm{~L}^{-1}$, modo DPV, amplitude $=100 \mathrm{mV}$, velocidade de varredura $10 \mathrm{mV} \cdot \mathrm{s}^{-1}$.

Figura 35 - Estudo do potencial de deposição 95

Figura 36 - Estudo da influência da amplitude sobre o sinal de oxidação da primaquina. Condições: primaquina $5,0 \times 10^{-3} \mathrm{~mol} \mathrm{~L}^{-1}$, tempo de deposição 0 $\mathrm{s}$, potencial inicial $0 \mathrm{~V}$, velocidade de varredura de $10 \mathrm{mV} \mathrm{s}^{-1}$. 97

Figura 37 - Influência da amplitude sobre a largura do pico a meia altura. $\quad 98$ Figura 38 - Influência da velocidade de varredura sobre o sinal de oxidação da primaquina.

Figura 39 - curva analítica para primaquina. 


\section{Lista de tabelas}

Tabela 1 - Estudo do eletrólito de suporte para o ciclofenil no EGPM. Condições experimentais: modo DPV; tempo de deposição $0 \mathrm{~s}$; velocidade de varredura $10 \mathrm{mV} / \mathrm{s}$; potencial inicial $0,1 \mathrm{~V}$; potencial final - 1,7 V; amplitude $100 \mathrm{mV}$.

Tabela 2 - Efeito do tempo de irradiação UV no sinal de uma solução de ciclofenil (quatro medidas subsequentes do fotoproduto do ciclofenil). Condições experimentais: concentração de ciclofenil $1,0 \times 10^{-6} \mathrm{~mol} \mathrm{~L}^{-1}$; tempo de deposição $30 \mathrm{~s}$; incremento $=10 \mathrm{mV}$; frequência $100 \mathrm{~Hz}$; amplitude $250 \mathrm{mV}$. 64

Tabela 3 - Resultado da recuperação do ciclofenil em medicamentos. $\quad 89$

Tabela 4 - Resultado da recuperação do ciclofenil em urina enriquecida 90

Tabela 5 - eletrólitos testados na tentativa de se obter sinal eletroanalítico da primaquina no EGPM. $\quad 91$

Tabela 6 - Resultado da recuperação da primaquina em urina enriquecida 


\section{Acronismos.}

DPV - Voltametria de pulso diferencial

AdSV - Voltametria adsortiva de redissolução

SWV - voltametria de onda quadrada

AAS - espectrometria de absorção atômica

HPLC - cromatografia líquida de alta eficiência

HPTLC - cromatografia líquida de camada delgada de alta eficiência

UA - unidades arbitrárias

ASV - Voltametria de redissolução anódica

CSV - Voltametria de redissolução catódica

TFE - Eletrodo de filme de mercúrio 